livraisons

d'Histoire

de l'Architecture

\section{Livraisons de l'histoire de l'architecture}

42 | 2021

2001-2021 / numéro anniversaire

\title{
Bamiyan, vingt ans après
}

Retour sur l'ambiguïté de l'émotion patrimoniale après la destruction des Bouddhas en 2001

Bamiyan, Twenty Years On. Reflecting on the Ambiguity of Heritage Emotion

After the Destruction of the Buddhas in 2001

Bamiyan, zwanzig Jahre danach. Ein Rückblick auf die Ambivalenz der

Emotionen zum Kulturerbe nach der Zerstörung der Buddhas im Jahr 2001

\section{Pauline Verger}

\section{OpenEdition}

\section{Journals}

Édition électronique

URL : https://journals.openedition.org//ha/3959

DOI : 10.4000/lha.3959

ISSN : 1960-5994

Éditeur

Association Livraisons d'histoire de l'architecture - LHA

Référence électronique

Pauline Verger, «Bamiyan, vingt ans après », Livraisons de l'histoire de l'architecture [En ligne], 42 | 2021, mis en ligne le 11 décembre 2021, consulté le 11 décembre 2021. URL : http:// journals.openedition.org//ha/3959; DOI : https://doi.org/10.4000//ha.3959

Ce document a été généré automatiquement le 11 décembre 2021.

Tous droits réservés à l'Association LHA 


\title{
Bamiyan, vingt ans après
}

Retour sur l'ambiguïté de l'émotion patrimoniale après la destruction des Bouddhas en 2001

\author{
Bamiyan, Twenty Years On. Reflecting on the Ambiguity of Heritage Emotion \\ After the Destruction of the Buddhas in 2001 \\ Bamiyan, zwanzig Jahre danach. Ein Rückblick auf die Ambivalenz der \\ Emotionen zum Kulturerbe nach der Zerstörung der Buddhas im Jahr 2001
}

Pauline Verger

En 2009 et 2011, les Livraisons d'histoire de l'architecture ont consacré deux numéros à l'étude des émotions patrimoniales, afin de comprendre comment certains monuments acquièrent une charge symbolique forte, qu'ils soient proches ou lointains, bien conservés ou en ruine. Par la force d'une image ou d'un événement, ces lieux catalysent les émotions; ils sont donc des révélateurs du regard que les sociétés portent sur le patrimoine, des représentations, des imaginaires, des mythes, des identités et des valeurs". Le terme "émotion patrimoniale", théorisé par Daniel Fabre dans les années 1990, désigne les émotions qui ont un point d'application particulier: «le patrimoine, le souci actuel de l'héritage venant du passé $»^{2}$. Les émotions patrimoniales peuvent être positives ou négatives, selon le contexte : admiration, nostalgie, haine, indignation ou désolation ${ }^{3}$. Elles naissent généralement du sentiment qu'un lieu ou un objet a de la valeur, qu'il doit être protégé, préservé et transmis aux générations futures. Patrimoine et émotions semblent ainsi aller de pair, à part peut-être pour les scientifiques et les experts qui privilégient une approche moins spontanée, plus standardisée. Pour le grand public, la relation au patrimoine n'est donc pas exempte d'émotions, du fait de la nature même du patrimoine, qu'on peut définir comme l'ensemble des traces du passé choisies parmi de nombreuses autres traces qui ont, elles, été laissées de côté. Le propos de cet article n'est pas d'analyser le processus de patrimonialisation, mais d'étudier la cristallisation des émotions à la fois individuelles et collectives autour des lieux, des objets et des pratiques reconnus comme patrimoine matériel ou immatériel. Les émotions patrimoniales, comme l'explique Nathalie Heinich, sont suscitées par un choc, le plus souvent un événement tragique qui 
marque les esprits comme un accident, un conflit ou une catastrophe naturelle. Parmi les événements récents, on peut citer, bien qu'ils aient eu des répercussions différentes, la destruction des Bouddhas de Bamiyan (2001), des manuscrits et des mausolées de Tombouctou (2012), de la cité antique de Palmyre (2015 et 2016), du minaret médiéval et de la grande mosquée al-Nouri de Mossoul (2017), et l'incendie de Notre-Dame de Paris (2019). À part ce dernier événement, les monuments cités ont été détruits volontairement, dans le cadre de conflits non-conventionnels, ou asymétriques, opposant des acteurs étatiques (armées professionnelles) à des acteurs non-étatiques (rebelles, groupes terroristes). La particularité de ces conflits, qui se multiplient depuis la fin du XXe siècle, est que les populations civiles sont non seulement touchées, ce qui est inévitable dans un conflit, quelle que soit sa nature, mais aussi directement ciblées, au mépris des règles classiques de la guerre inscrites dans le droit international humanitaire. Dans les conflits contemporains, les civils sont attaqués physiquement, par la destruction des moyens de subsistance, d'écoles, d'hôpitaux, par la réduction en esclavage, les viols, l'exécution d'otages, les génocides et, symboliquement, par la destruction de ce qui est considéré comme l'emblème de l'identité d'un groupe, c'est-àdire le patrimoine culturel. Pour autant, rien n'est nouveau, car les civils et le patrimoine culturel ont toujours souffert des conflits. Alors, pourquoi ce regain d'intérêt, ces émotions patrimoniales fortement exprimées dans les médias ${ }^{4}$ ? On peut avancer trois éléments de réponse : la multiplication des conflits dans le monde, la montée du fondamentalisme religieux et les avancées technologiques qui permettent de partager en temps réel des images dans le monde entier ${ }^{5}$. La destruction des Bouddhas de Bamiyan est à ce titre un moment décisif, un tournant, car il s'agit de la première destruction volontaire dont les images ont été diffusées très rapidement à l'échelle mondiale. Les talibans n'ont pas seulement détruit deux immenses statues de Bouddha, ils ont aussi photographié et filmé la destruction, diffusé les images dans les médias et invité les journalistes présents à Kaboul à attester de la destruction.

2 En 2009, les Livraisons d'histoire de l'architecture publiaient dans le numéro consacré aux émotions patrimoniales un article de l'ethnologue Pierre Centlivres intitulé «Vie, mort et survie des Bouddhas de Bamiyan ». L'auteur expliquait vouloir analyser, «par-delà les événements factuels, les enjeux et les valeurs exprimés dans l'argumentation des parties en cause : les bouddhistes, la population afghane et le public occidental ». Vingt ans après la destruction de Bamiyan, il nous paraît intéressant de réfléchir à la manière dont est commémoré un tel événement, en confrontant les valeurs, les discours et les représentations, pour étudier comment la destruction a été montrée dans les médias occidentaux, comment a évolué l'intensité de l'émotion patrimoniale, et comment le patrimoine a été politisé, instrumentalisé, utilisé comme une arme de guerre. Cet article étudiera donc, du point de vue occidental, la fabrique de l'émotion patrimoniale après la destruction des Bouddhas de Bamiyan.

3 La première question posée est : que s'est-il passé à Bamiyan? Quel est le sens de cette destruction spectaculaire? Était-ce un défi, un acte de colère, un accès de folie, une déclaration de guerre, une affirmation d'autorité, une manière d'asseoir un pouvoir ? La deuxième question interroge le sens de l'émotion patrimoniale : à quelle échelle, selon quelle temporalité s'est manifestée l'émotion? Y a-t-il eu consensus dans l'indignation et la condamnation de l'iconoclasme? Aujourd'hui, avec la distance dans le temps et dans l'espace, et la multiplication de tels actes de destruction dans l'actualité, l'émotion est-elle amoindrie, dispersée ? Enfin, la dernière question porte 
sur l'avenir du site : au-delà de l'émotion patrimoniale, quels sont les enjeux de la protection des vestiges archéologiques de la vallée de Bamiyan?

4 Il est à noter que cet article a été écrit avant le retour au pouvoir des talibans en Afghanistan et la prise de Kaboul le 15 août 2021. Il a été réactualisé depuis, mais les relations de pouvoir décrites ici sont susceptibles d'être profondément bouleversées et remaniées, avec par exemple l'apparition de nouveaux acteurs tels que la branche afghane de Daech.

\section{Bamiyan, mars 2001 : un spectacle à grande échelle pour une émotion mondiale}

\section{Le site}

5 Jusqu'en 2001, la vallée de Bamiyan, située au centre de l'Afghanistan à 2500 mètres d'altitude, abritait deux immenses statues de Bouddha taillées dans la roche, hautes de 38 et 55 mètres. Aujourd'hui il ne reste que les niches des statues. Tout autour, des centaines de grottes décorées de fresques forment un ensemble de monastères, de sanctuaires et de chapelles bouddhistes datant $\mathrm{du}_{\mathrm{III}}^{\mathrm{e}}$ au V $\mathrm{V}^{\mathrm{e}}$ siècle. Dans la vallée de Kakrak, à quelques kilomètres au sud-est de Bamiyan, une autre statue de Bouddha, de 10 mètres de haut, est sculptée dans la falaise. De nombreuses sources historiques donnent une description précise du site avant sa destruction: des témoignages de voyageurs, des photographies et des archives archéologiques. En 632, le pèlerin bouddhiste chinois Xuanzang s'arrêta à Bamiyan au cours de son voyage vers l'Inde. Il raconta ses souvenirs dans son Rapport du voyage en Occident à l'époque des Grands Tangs. Ce récit de voyage est le plus ancien texte dont on dispose qui mentionne l'existence des monastères bouddhistes et des statues de Bamiyan :

6 "Sur le flanc d'une montagne située au nord-est de la ville royale, il y a une statue en pierre du Bouddha qu'on a représenté debout; elle est haute de cent quarante à cent cinquante pieds. Elle est d'une couleur d'or qui rayonne de toutes parts, et l'œil est ébloui de ses précieux ornements $»^{6}$.

7 Dans son récit, Xuanzang décrit les deux statues de Bouddha et mentionne également une immense statue de Bouddha en nirvana que les archéologues n'ont toujours pas retrouvée. Le site de Bamiyan a été découvert par les Occidentaux au XIX ${ }^{\mathrm{e}}$ siècle, à l'époque où l'Asie centrale était au cœur des tensions entre la Grande-Bretagne et la Russie, dans le cadre du Great Game ${ }^{7}$, c'est-à-dire des rivalités coloniales entre les deux empires en expansion. Dans ce contexte, comme le rappelle Pierre Centlivres ${ }^{8}$, les premiers voyageurs occidentaux en Afghanistan furent des espions, des militaires et des trafiquants de toutes sortes. En 1832, Alexander Burnes ${ }^{9}$ fit le croquis des deux statues qui représentaient selon lui un homme et une femme, nommés Salsal et Shah Mama (Ill. 1). Il fut suivi de Charles Masson en 1835 et du capitaine Maitland en 1886. $\mathrm{Au} \mathrm{XX}^{\mathrm{e}}$ siècle, Bamiyan fut visité par des écrivains-voyageurs comme Robert Byron et Ella Maillart. Le premier ne fut guère impressionné par les statues, mais la seconde fut très sensible à la beauté du site dans son ensemble :

«Baigné de cette atmosphère de souriant repos, le paysage est ce qu'il y a de plus bienfaisant à Bamiyan. C'est probablement la raison pour laquelle des bouddhistes vinrent ici établir des monastères au début de notre ère $»^{10}$. 


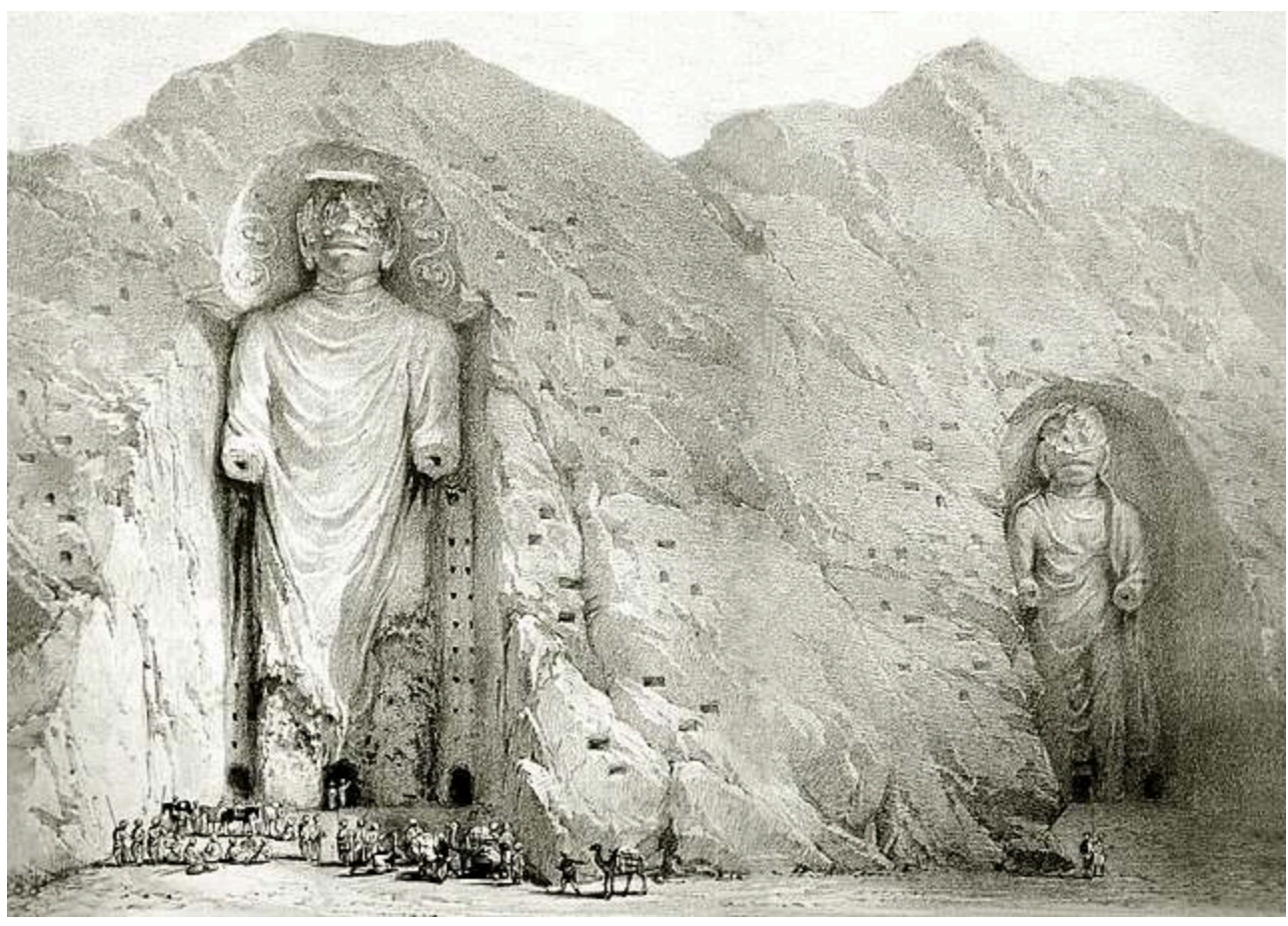

Gravure de L. Haghe tirée des Travels into Bokhara d'Alexander Burnes, London, 1834.

Source Wikimedia - domaine public.

8 Le site fut fouillé par les archéologues dès 1922, c'est-à-dire dès la création de la Délégation archéologique française en Afghanistan (DAFA) à l'initiative du roi Amanullah. Dans leurs comptes-rendus, les archéologues français expliquent que Bamiyan, autrefois une étape importante sur les routes de la soie, était devenu un passage obligé pour les voyageurs, qu'ils soient touristes, membres de l'expédition Citroën de 1931, archéologues, ou, plus tard, hippies en route vers Katmandou dans les années 1970 :

"Situé sur une grande route du commerce et de la guerre, marqué par des vestiges grandioses et singuliers, signalé par l'admiration des pèlerins chinois, ce lieu célèbre s'imposait des premiers à l'active curiosité des nouveaux venus $»^{11}$.

Les Bouddhas étaient donc un élément emblématique de la vallée de Bamiyan et même, aux yeux des voyageurs occidentaux, de l'Afghanistan tout entier. Il est plus difficile de savoir ce qu'ils représentaient aux yeux des Afghans. Alexander Burnes avait remarqué que les habitants de la vallée voyaient dans les statues un homme et une femme, un couple d'idoles parfois assimilé à des déesses mentionnées dans le Coran. La poste afghane émit des timbres à l'effigie du grand Bouddha dans les années 1930, mais, après les vives protestations de responsables religieux, ils furent retirés de la circulation.

\section{La destruction}

10 La région de Bamiyan, le Hazarajat, n'eut pas à souffrir des combats pendant la guerre contre l'URSS (1979-1989). Dans les années 1990, pendant la guerre civile qui suivit le 
retrait des Soviétiques, le Hazarajat obtint plus d'autonomie. Les hommes politiques et les intellectuels hazaras s'appuyèrent sur " un mythe ethnique " pour se différencier des autres régions et populations afghanes ${ }^{12}$. Les Bouddhas de Bamiyan devinrent alors le symbole de l'identité hazara, certains intellectuels affirmant que les statues représentaient les ancêtres de la population hazara.

11 Après la chute du gouvernement communiste en 1992, les factions rivales se disputèrent le pouvoir. Les talibans montèrent en puissance en 1994-1995. Ils affirmaient vouloir libérer l'Afghanistan de la corruption, de la guerre, de la pauvreté, en mettant en place une société islamique pure et respectueuse de la charia. Le mouvement taliban obtint au début le soutien de la population afghane épuisée par la guerre civile et les combats entre les différents seigneurs de guerre. En septembre 1996, les talibans s'emparèrent de Kaboul. Deux ans après, ils s'emparèrent de Bamiyan. À partir de ce moment-là, des chefs talibans commencèrent à détruire les Bouddhas, de leur propre initiative. En mai 1999, la newsletter d'une organisation nongouvernementale dédiée à la protection du patrimoine afghan, SPACH (Society for the Preservation of Afghanistan's Cultural Heritage) annonça, preuves photographiques à l'appui, que le petit Bouddha avait subi de nombreux dommages: sa tête avait été bombardée et son corps pris pour cible lors d'entraînements au tir ; il avait de larges trous au niveau du bras gauche et à l'aine; le drapé de stuc tombait peu à peu; et les fresques qui ornaient la niche au-dessus de la tête du Bouddha avaient disparu ${ }^{13}$. En juillet 1999, le ministère de la culture des talibans garantit par décret la protection des antiquités afghanes. Or, moins de deux ans plus tard, le 26 février 2001, Mullah Omar, chef des talibans, décréta que le patrimoine préislamique afghan devait être détruit. Pourquoi ce revirement? Les logiques internes au mouvement des talibans peuvent expliquer ces contradictions. Loin d'être homogène, le mouvement est marqué par les tensions entre ceux qui sont dits "durs", "conservateurs", et ceux qui sont " modérés ", "éclairés » ${ }^{14}$. L'iconoclasme de Bamiyan a donc une explication religieuse et idéologique que l'on retrouve dans le décret du 26 février 2001 :

«Toutes les statues et les sanctuaires non islamiques sis dans les différentes parties de l'émirat [d'Afghanistan] doivent être détruits. Ces statues ont été et restent des sanctuaires d'infidèles, et ces infidèles continuent à adorer et à vénérer ces images. Allah Tout-Puissant est le seul vrai sanctuaire, et tous les faux symboles doivent être fracassés, afin que personne ne puisse les adorer ou les vénérer à l'avenir $»^{15}$.

Toutefois cette déclaration ne suffit pas à expliquer le revirement des talibans. La destruction spectaculaire est ainsi à comprendre dans le cadre du contexte politique international. Depuis qu'ils avaient proclamé l'émirat islamique d'Afghanistan en 1996, les talibans cherchaient à obtenir la reconnaissance internationale de leur régime. Le décret de juillet 1999 était un gage de bonne volonté, montrant que les talibans étaient prêts à coopérer avec l'UNESCO ou l'organisation non-gouvernementale SPACH. Mais les organisations internationales, compte tenu du non-respect du droit international, des nombreuses violations des droits de l'Homme et de la présence d'Oussama Ben Laden ${ }^{16}$ sur le territoire afghan, prirent le 16 octobre 1999 des sanctions contre l'émirat islamique d'Afghanistan. Ces sanctions entrèrent en vigueur le 19 janvier 2001. Le décret du 26 février 2001 fut alors peut-être un acte de colère, Mullah Omar n'ayant obtenu ni la reconnaissance internationale ni les aides financières demandées. Comment furent détruits les Bouddhas? Les statues furent « attaquées» militairement 
durant la première semaine de mars $2001^{17}$, à grand renfort de moyens pyrotechniques, d'artillerie et d'explosifs. Le petit Bouddha fut dynamité le 9 mars, et le grand Bouddha les 10 et 11 mars. Le 16 mars, Mullah Omar ordonna le sacrifice de cent vaches pour expier le retard pris dans la destruction des statues ${ }^{18}$. Le 18 mars, une vidéo montrant l'explosion du petit Bouddha fut diffusée sur Al-Jazeera et CNN. Enfin, le 26 mars, les journalistes étrangers présents à Kaboul furent invités à constater la disparition des Bouddhas. Interviewé par une journaliste du Monde, un combattant taliban raconta :

«Nous avons tout fait sauter, il y a dix jours. Nous avons commencé par les jambes du grand Bouddha, puis nous avons détruit le petit. Il nous a fallu quatre jours pour venir à bout du grand Bouddha [avec] des mines, des explosifs et même des obus de chars. Nous sommes très heureux $»^{19}$.

Cette visite guidée organisée le 26 mars 2001 pour une vingtaine de journalistes étrangers manifeste une volonté évidente de mise en scène de la part des talibans.

\section{La mise en scène des talibans}

La chronologie de la destruction des Bouddhas de Bamiyan est bien connue. Elle n'a pas manqué de choquer l'opinion publique occidentale particulièrement ciblée par les talibans. En effet, la destruction des Bouddhas fut un véritable spectacle que l'on pourrait qualifier, dans un autre registre, de coup publicitaire, de happening; un spectacle, donc, avec sa scène, son public, ses acteurs, ses figurants et sa campagne de publicité.

15 La scène, d'abord. La vallée de Bamiyan, avec ses Bouddhas géants sculptés dans la falaise et ses centaines de grottes, est un lieu grandiose, photogénique s'il en est, bien connu des voyageurs occidentaux et des archéologues. C'est l'un des lieux qui, aux yeux des étrangers, incarne l'Afghanistan. Dans les années 1980, quatre sites afghans, dont Bamiyan, avaient d'ailleurs été inscrits sur la liste indicative de l'UNESCO.

Les talibans furent à la fois acteurs et metteurs en scène. Du début à la fin, ils eurent l'initiative des discours et des actes, réduisant à l'état de figurants les habitants de la vallée de Bamiyan. On ne vit qu'eux, on n'entendit qu'eux dans la manière dont la presse occidentale traita les événements; la population locale ne fut guère évoquée, comme le montrent, par exemple, les titres des articles que le journal Le Monde publia au sujet de Bamiyan du 27 février au 27 mars 2001 : quatorze des dix-sept articles au sujet de Bamiyan mentionnaient les talibans dans leur titre, alors que les Hazaras ne furent jamais présentés au premier plan. Les talibans occupèrent donc le devant de la scène médiatique en mars 2001.

Le public fut à la fois la population afghane, la communauté internationale, les sociétés occidentales et les bouddhistes. Les talibans firent preuve d'une certaine maîtrise de la communication, par leurs déclarations officielles, l'incertitude (allaient-ils vraiment détruire les statues ou pas?), puis la diffusion du film de la destruction du petit Bouddha et l'invitation des journalistes étrangers à Bamiyan.

18 La multiplication des montages photographiques "avant/après » la destruction nous renseigne sur ce pouvoir de l'image que semblent avoir très bien compris les talibans : les niches des Bouddhas ressemblent aujourd'hui à des sarcophages vides, des trous béants dans la falaise qui interpellent et marquent fortement les esprits (Ill.2). Paradoxalement, les talibans, qui voulaient attaquer des idoles, ont mis en scène 
l'image de la destruction des images; ils ont voulu marquer un grand coup, susciter l'émotion patrimoniale, mais pourquoi?

\section{2. Le grand Bouddha avant et après la destruction}

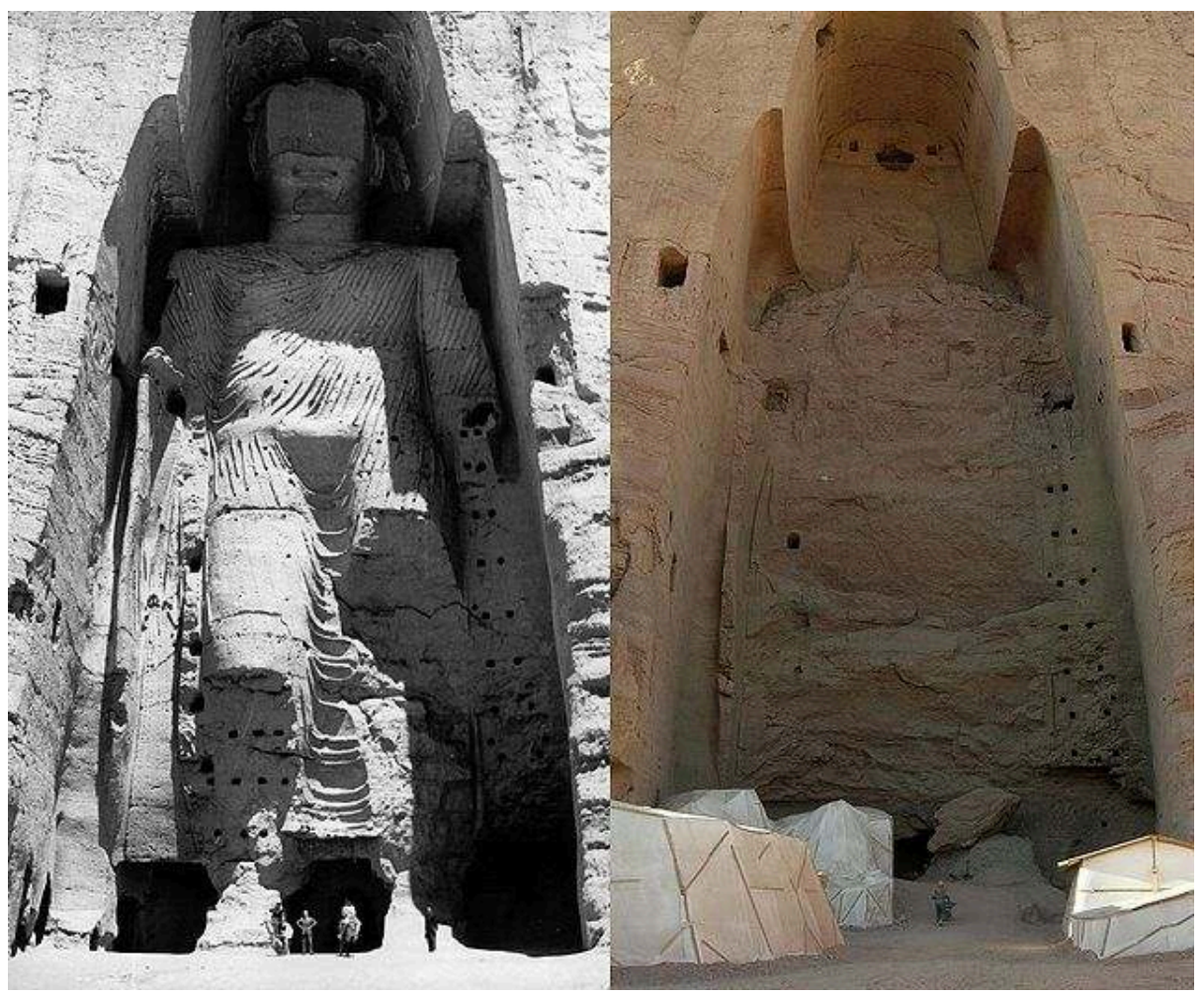

Montage photographique d'après une photographie de 1963 par l'UNESCO/A. Lezine et une photographie de 2008 par Carl Montgomery, 2009.

Source Wikimedia - CC BY-SA 3.0

\section{Émotion, émotions}

\section{Hier}

19 Quels étaient donc les buts des talibans? Pourquoi ont-ils instrumentalisé le patrimoine et fait naître l'émotion à l'échelle internationale, nationale et locale?

À l'échelle internationale, les Nations Unies, les chefs d'État, les médias et les personnalités du monde de la culture ont unanimement condamné l'iconoclasme des talibans, associé à l'idéologie islamiste. Dépêché en urgence, le représentant spécial de l'UNESCO en Afghanistan, Pierre Lafrance, échoua à convaincre les talibans de renoncer à la destruction des Bouddhas. Il expliqua rétrospectivement :

"On savait, il est vrai, que le mouvement ayant imposé sa loi à Kaboul entendait réduire la piété islamique dont il se réclamait à un ensemble de règles simples : faire la prière aux heures dites, cloîtrer les femmes, couper la main des voleurs, user de la loi du talion, détruire les statues, interdire les images $»^{20}$.

21 Grâce aux technologies permettant de filmer et de diffuser rapidement les images de Bamiyan, la destruction provoqua une vague d'émotion mondiale reflétée dans les 
grands titres de la presse. À cette échelle, la destruction des Bouddhas fut semblable à un coup publicitaire destiné à montrer que les talibans pouvaient défier la communauté internationale qui refusait de reconnaître la légitimité de leur régime.

À l'échelle nationale, la destruction fut une manière de légitimer le pouvoir des talibans après des années de conflit, ou du moins de montrer qu'ils étaient les plus forts. Les talibans eurent beau jeu de dénoncer l'hypocrisie des occidentaux mobilisés pour sauver de vieilles pierres alors que des enfants mouraient de faim. C'est le discours qu'ils tinrent dans les médias, par exemple dans le New York Times le 19 mars 2001 :

"When your children are dying in front of you, then you don't care about a piece of art. [...] If you are destroying our future with economic sanctions, you can't care about our heritage. [...] If we had wanted to destroy those statues, we could have done it three years ago. So why didn't we? In our religion, if anything is harmless, we just leave it. If money is going to statues while children are dying of malnutrition next door, then that makes it harmful, and we destroy it" ${ }^{21}$.

Selon cette argumentation, toutes les démarches pour sauver les bouddhas, les propositions d'achat ou de déplacement n'auraient fait qu'augmenter la colère des talibans choqués par une telle indécence.

À l'échelle locale, la population hazara fut directement visée par la destruction des Bouddhas qui étaient le symbole de la vallée. Les médias occidentaux ne parlèrent pas des habitants de Bamiyan en 2001. On ne sait donc pas comment ils ont réagi, ni quel était leur degré d'attachement aux statues. Toutefois, en 2015, un reportage de la BBC donna la parole à un ancien prisonnier hazara, Mirza Hussain, qui avait été forcé par les talibans à participer à la destruction des Bouddhas :

"I regretted it at that time, I regret it now and I will always regret it. But I could not resist, I didn't have a choice because they would have killed me"22.

En 2011, dix ans après la destruction, un reportage de l'OTAN avait interrogé les habitants de Bamiyan. Ceux-ci avaient expliqué qu'ils admiraient les statues et que la vallée avait perdu une source de revenus conséquente car beaucoup de touristes venaient voir les Bouddhas. Ces témoignages demeurent cependant assez rares dans les médias occidentaux, parce que l'indignation suscitée par la destruction des Bouddhas s'accompagna d'un phénomène d'appropriation. Puisque le site était considéré par l'UNESCO comme l'un des symboles du patrimoine culturel mondial, l'humanité tout entière était touchée par sa disparition, ce qui eut pour conséquence de reléguer les Hazaras, et plus largement les Afghans, au second plan.

\section{Aujourd'hui}

Les deux grandes niches privées de leurs Bouddhas sont devenues des « ruines traumatiques ${ }^{23}$ autour desquelles s'organisent commémorations et résilience. Les grands anniversaires, un an en 2002, dix ans en 2011, vingt ans en 2021, sont l'occasion d'exprimer l'émotion patrimoniale dans les sociétés occidentales. En dehors de ces grandes dates, les Bouddhas de Bamiyan sont régulièrement évoqués dans les médias. En 2002, le musée de la Caixa à Barcelone puis le musée Guimet à Paris consacrèrent une exposition au patrimoine afghan. Le journal Libération expliqua à cette occasion que le musée Guimet était devenu une sorte de musée de Kaboul en exil ${ }^{24}$. Une autre 
exposition itinérante ${ }^{25}$ voyagea entre 2008 et 2015 en Italie, en Angleterre, aux PaysBas, en Norvège, aux États-Unis et en Australie. À l'occasion des quinze ans de la destruction ; en 2016, France Culture consacra une série de trois podcasts sur « Ce que voient les Bouddhas ». En 2017, une journée " Mémoire de Bamiyan» fut organisée au Palais de Tokyo à Paris en partenariat avec France Culture, l'Institut français et l'Ambassade de France en Afghanistan. Enfin, en 2021, les médias occidentaux commémorèrent les vingt ans de la destruction, comme le montrent quelques titres : "Afghanistan : il y a vingt ans, la destruction des Bouddhas de Bamiyan " (France 24), « Destruction des Bouddhas de Bamiyan : vingt ans déjà » (RFI), « In pictures : 3D return for Bamiyan Buddha destroyed by Taliban » $(\mathrm{BBC})$, « 20 años de la destrucción de los budas de Bamiyan» (El Tiempo, 9 mars). Un communiqué de l'UNESCO, le 11 mars, rappela que la destruction des Bouddhas avait « conduit à une reconnaissance mondiale de la nécessité de protéger le patrimoine culturel en péril». En France, deux expositions furent organisées, l'une au Musée Guimet («Des images et des hommes, Bamiyan, 20 ans après ») et au Louvre-Lens («Bamiyan, la falaise et la grotte »). L'organisation non-gouvernementale Patrimoine sans frontières consacra quant à elle une série d'articles sur le patrimoine afghan.

Les titres des articles et les événements organisés pour commémorer la destruction montrent l'adoption progressive d'une vision plus inclusive du patrimoine. En 2001, les médias s'étaient concentrés sur les statues, c'est-à-dire sur le patrimoine matériel, sans vraiment évoquer ce qu'elles représentaient pour la population locale; en 2021, il semble que le patrimoine est désormais compris dans sa dimension matérielle et dans sa dimension immatérielle, l'UNESCO soulignant l'importance de la "tradition culturelle d'Asie centrale » incarnée à Bamiyan. Le 9 mars 2021 fut organisée à Bamiyan une journée de commémoration conclue par une "Nuit avec Bouddha " au cours de laquelle l'un des Bouddhas réapparut sous la forme d'un hologramme à l'intérieur de son alcôve. D'après la presse, des centaines de personnes se rassemblèrent en procession, une lanterne à la main, pour admirer la reconstitution éphémère du grand Bouddha $^{26}$. Les falaises de Bamiyan sont des « ruines traumatiques » parce que la vision des niches vides est frappante, mais aussi parce que le film de la destruction a été largement diffusé. Il est aujourd'hui disponible sur Youtube, à la portée de tous, sans aucun avertissement préliminaire. La manière dont la destruction est filmée donne l'impression d'être sur place, à Bamiyan : on voit clairement le Bouddha disparaître sous une épaisse fumée noire, on entend les talibans parler et crier, et l'onde de choc déplace la caméra. Il s'agit d'un exemple canonique d'instrumentalisation du « pouvoir de l'image $»^{27}$.

\section{Paradoxes et ambiguiités}

28 L'apparente unanimité de l'émotion patrimoniale après la destruction des Bouddhas de Bamiyan cache un certain nombre de questionnements éthiques, politiques et scientifiques. L'émotion patrimoniale n'est pas exempte de biais et de paradoxes au premier rang desquels figure ce qu'on pourrait appeler le paradoxe de la concomitance : c'est au moment même où les Bouddhas disparurent que le grand public occidental découvrit leur existence. De plus, s'ils étaient bien connus des voyageurs et des scientifiques, les Bouddhas doivent leur notoriété actuelle à l'iconoclasme des talibans ; ils sont encore plus célèbres maintenant qu'ils ne sont plus là : c'est ce qu'on pourrait appeler le paradoxe de l'absence. 
Après les attentats du 11 septembre 2001, le rapprochement fut fait avec les images de la destruction de Bamiyan. En même temps, un intérêt nouveau pour l'Afghanistan se développa, à la fois pour les chercheurs et pour le grand public: " On September 11, 2001, Afghanistan suddenly became relevant ${ }^{28}$ ». Les chercheurs en security studies américains investirent massivement le sujet à tel point qu'il devint difficile d'étudier l'Afghanistan autrement que sous l'angle de la lutte antiterroriste et des politiques de sécurité. Vingt ans après, alors que l'armée américaine s'est retirée d'Afghanistan et que les talibans ont repris le pouvoir, on peut se demander qui a créé et alimenté l'émotion patrimoniale. À première vue, ce sont les talibans, puisqu'ils ont donné à voir la destruction des Bouddhas au monde entier. Mais ne peut-on pas considérer que les médias occidentaux ont, eux aussi, fabriqué l'émotion patrimoniale, en relayant les images des talibans, en les commentant, en mobilisant un registre pathétique et hyperbolique ? Le traitement de la destruction des Bouddhas dans les médias a informé le public, au sens étymologique : il a façonné ses réactions et ses émotions. En quelque sorte il a fait le jeu des talibans. On pourrait objecter à cet argument que l'information est le propre des médias; mais il existe des moyens d'informer le public sans pour autant mettre les terroristes au premier plan. C'est par exemple la politique de la première ministre néo-zélandaise Jacinda Ardern, qui refusa de citer le nom du tueur après les attentats de Christchurch en mars 2019, pour laisser toute la place aux victimes. Dans le cas de Bamiyan, il n'y a pas eu mort d'homme, et c'est donc symboliquement que la question des victimes s'est posée. Qui a été (le plus) touché par la destruction des Bouddhas de Bamiyan : les Hazaras? les Afghans? les bouddhistes? les archéologues ? l'UNESCO ? l'humanité ?

L'émotion patrimoniale n'a pas forcément été consensuelle, puisqu'elle est à la fois individuelle et collective, véhiculée, amplifiée par les médias, mais aussi dépendante de la sensibilité de chacun. C'est pourquoi le pluriel "émotions patrimoniales» nous paraît en définitive plus juste.

\section{Bamiyan, mars 2021 : au-delà des émotions patrimoniales}

\section{La destruction : cause immédiate et causes profondes}

31 Après le temps de l'émotion vient le temps de la réflexion. Dans son histoire de la guerre du Péloponnèse, Thucydide distingue la cause immédiate et la cause profonde du conflit qui opposa Athènes à Sparte. Afin de dépasser le stade de la haine patrimoniale d'un côté, et de l'indignation de l'autre côté, suivons la méthode de Thucydide pour analyser les causes de la destruction de Bamiyan à court, moyen et long terme.

32 À court terme, en mars 2001, les Talibans étaient isolés sur la scène internationale et ne pouvaient pas prouver leur légitimité selon les règles du droit international. De plus, ils dénonçaient constamment le mode de vie occidental. Ils étaient donc un groupe relativement faible face à l'ensemble de la communauté internationale, et aux ÉtatsUnis en particulier. Confrontés directement à une telle puissance, ils n'auraient jamais pu gagner. Mais, symboliquement, ils pouvaient attaquer ce qu'ils savaient avoir de la valeur pour la communauté internationale. En un sens, le patrimoine fut pris en otage par les talibans. Ensuite, il fut utilisé comme une arme contre les Hazaras. Les talibans 
étaient donc en position de faiblesse par rapport aux États-Unis, mais en position de supériorité en Afghanistan. Ils ont alors manipulé les représentations et les valeurs pour influencer en leur faveur les rapports de pouvoir. À moyen terme, les décennies de conflit en Afghanistan expliquent pourquoi le patrimoine est si menacé, et pas seulement à Bamiyan: l'invasion soviétique, la guerre civile, le conflit entre les moudjahidines et les talibans ont transformé le pays en un État failli. Quand des vies humaines sont en danger, il est difficile de protéger les monuments et les œuvres d'art. Le musée de Kaboul fut attaqué et pillé plusieurs fois. Encore aujourd'hui, les sites archéologiques sont pillés lors de fouilles sauvages. Depuis la reprise de Kaboul par les talibans le 15 août 2021, il est difficile d'avoir des informations fiables sur les sites patrimoniaux, mais aucun ne semble avoir été visé intentionnellement comme Bamiyan l'a été en 2001. À long terme, des tensions idéologiques et historiographiques peuvent expliquer l'iconoclasme des talibans. On a dit que Bamiyan est un haut lieu de l'archéologie en Afghanistan. En effet, c'est là que les archéologues de la DAFA firent leurs premières fouilles en 1922. Bamiyan incarne le croisement des cultures et des arts, des influences hellénistique, bouddhiste, iranienne et indienne. Les deux statues étaient emblématiques de l'art gréco-bouddhique, aussi appelé art du Gandhara: le réalisme du drapé rappelait la statuaire grecque tandis que le sujet représenté était d'inspiration orientale (Ill. 3). Longtemps les archéologues occidentaux ont considéré que l'art gréco-bouddhique était plus intéressant à étudier que l'art islamique. Daniel Schlumberger, directeur de la DAFA de 1946 à 1964 expliqua ainsi lors d'une conférence à la Maison de France de Kaboul en 1964 :

«Le passé de l'Afghanistan, du point de vue de l'histoire de la civilisation, se présente comme un diptyque. L'un des volets de ce diptyque, le volet antique, est hellénique; l'autre volet, le volet médiéval et moderne, est islamique. [...] On pense en grec, on écrit en grec à Kandahar, au troisième siècle avant $\mathrm{J}$.-C., exactement comme on ferait à Athènes ou à Milet $»^{29}$. 


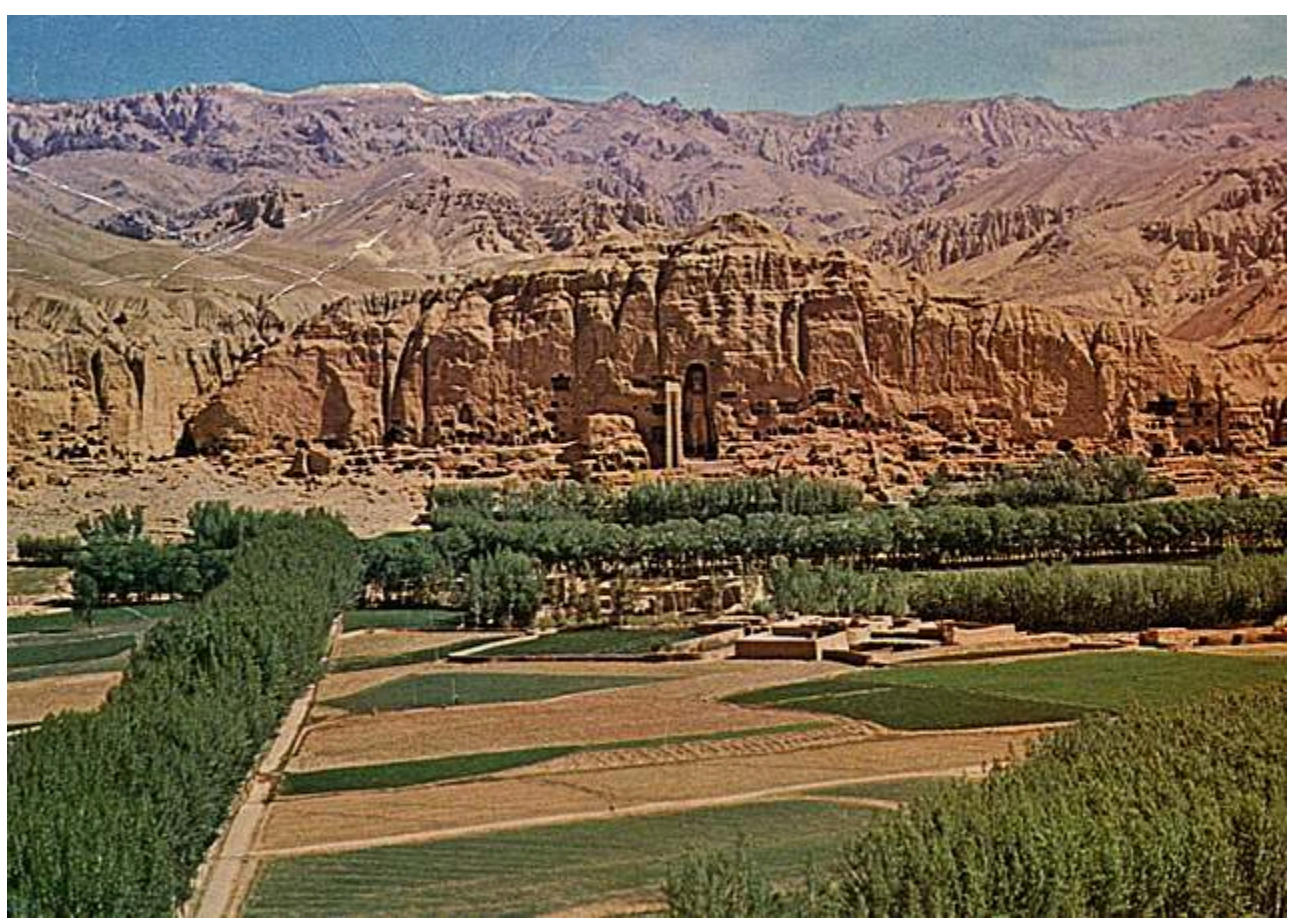

1967, carte postale.

Source Wikimedia - CC BY-SA 3.0 igo

33 L'archéologie rejoignait la politique puisqu'au même moment, les archéologues afghans tels qu'Ahmad Ali Kuhzad tentaient d'unir le pays autour d'un passé préislamique glorieux, marqué par le passage d'Alexandre le Grand en Afghanistan. Cette dichotomie entre histoire antique et histoire moderne explique pourquoi les sites islamiques furent fouillés seulement après la Seconde guerre mondiale. La destruction des Bouddhas s'inscrit pleinement dans ces tensions historiographiques: ce fut un acte politique de négation d'une certaine interprétation, et appropriation, de l'histoire de l'Afghanistan.

\section{La protection du patrimoine afghan : controverses théoriques}

La vague d'émotions patrimoniales après la destruction des Bouddhas alimente les controverses portant sur les notions de valeur et de patrimoine. Comment définir ce qui a de la valeur, et pour qui, pour quel public, pour quelle population? Qui est juge de la valeur? Selon quels critères? L'UNESCO a classé deux sites afghans, la vallée de Bamiyan et le minaret de Djam, sur sa liste du patrimoine mondial. Cette liste suppose que la valeur des sites classés est universellement reconnue et acceptée :

«Ce qui rend exceptionnel le concept de patrimoine mondial est son application universelle. Les sites du patrimoine mondial appartiennent à tous les peuples du monde, sans tenir compte du territoire sur lequel ils sont situés $»^{30}$.

Or, la notion de patrimoine est culturellement datée. Enracinée dans l'histoire européenne, elle a progressivement évolué, passant du domaine privé au domaine public, ne se limitant plus à l'art sacré et devenant peu à peu une "valeur laïque ${ }^{31}$ qui a pu sembler suspecte aux talibans. Par ailleurs, la convention de l'UNESCO portant sur 
la protection du patrimoine mondial (1972) est marquée, dans sa version anglaise, par une autre ambiguïté : le patrimoine y est désigné par deux termes, « cultural heritage " et «cultural property». Or, " property» se traduit aussi par propriété, ce qui pose la question d'une éventuelle appropriation du patrimoine. Comme l'ont montré de nombreux articles de presse, les Occidentaux ont porté le deuil des statues comme si elles leur appartenaient, sans se demander ce que pensaient les Afghans de la destruction. Pour Pierre Centlivres ${ }^{32}$, ces émotions occidentales expliquent pourquoi les talibans ont pris en otage les Bouddhas : d'un côté, le trop-plein d'émotions pour des statues; de l'autre côté, l'absence d'émotions pour des êtres vivants. L'enjeu est donc de trouver une manière de condamner la destruction du patrimoine sans pour autant se l'approprier. Cette alternative aux revendications occidentales de propriété culturelle doit être enracinée dans des critiques locales des talibans, privilégiant les voix hazaras ou afghanes.

\section{La protection du patrimoine afghan : enjeux pratiques}

Le 4 janvier 2004, à la $105^{\mathrm{e}}$ réunion annuelle de l'Institut archéologique d'Amérique, Abdul Wasey Feroozi ${ }^{33}$, directeur de l'Institut afghan d'archéologie, donna une conférence sur les conséquences de la guerre sur le patrimoine afghan. Après avoir expliqué que «la catastrophe de la guerre [avait] anéanti soixante-dix ans de dur labeur et de réussites ", il supplia la communauté scientifique et le grand public de ne pas laisser retomber l'émotion patrimoniale suscitée par la destruction des Bouddhas de Bamiyan et de rester mobilisé pour « ne pas oublier l'Afghanistan ». Il soulignait par là un des biais de l'émotion patrimoniale qui, comme toute émotion, est fugace. Vingt ans après, que reste-t-il de l'émotion patrimoniale causée par les talibans à Bamiyan ? L'anniversaire de la destruction a été commémoré, on l'a vu, dans les médias occidentaux. Mais il semble que ce soit devenu un souvenir lointain, souvent cité, mais pas vraiment connu des jeunes générations.

Les fouilles archéologiques ont repris dès 2002 à Bamiyan, en parallèle des opérations de sauvegarde de ce qui n'avait pas été détruit. La falaise, en particulier, avait besoin d'être consolidée. Des missions archéologiques internationales ont travaillé sur place, ainsi que des organisations non-gouvernementales. Le site a dû être déminé, et des agents de sécurité ont été engagés pour en assurer la protection. En 2011, des discussions ont été engagées pour retirer Bamiyan de la liste du patrimoine mondial en péril, mais à ce jour, cette démarche n'a pas abouti. Des projets de reconstruction ont été évoqués, mais rien n'a été entrepris pour l'instant. Le retour des talibans au pouvoir renforce cette incertitude quant à l'avenir du site. La destruction des Bouddhas de Bamiyan a occulté les autres menaces qui pèsent sur le patrimoine afghan. Les monuments et les musées ont souffert des dommages collatéraux de la guerre: monuments détruits, terrains minés, insécurité, manque de moyens... Mais la plus grande menace est actuellement le pillage des sites et des musées. Selon Omar Khan Massoudi, directeur du musée de Kaboul, environ $70 \%$ des collections du musée ont été volés durant les années de conflit pour être revendus au marché des antiquités de Peshawar, au Pakistan ${ }^{34}$. Les sites archéologiques ont été massivement pillés, et le sont encore de nos jours; certains avaient déjà été fouillés par les archéologues, d'autres non, ce qui représente une perte immense pour la science ${ }^{35}$. Paradoxalement, les collections archéologiques afghanes ont longtemps été plus en sécurité à l'étranger, au musée Guimet en particulier, avec les collections de la 
Délégation archéologique française en Afghanistan. Depuis sa publication en 1982, l'Archaeological Gazetteer of Afghanistan ${ }^{36}$ de Warwick Ball est un ouvrage de référence listant les grands sites et monuments de la région. Or, comme le déplora Abdul Wasey Feroozi en $2004^{37}$, cette liste détaillée de près de 2800 sites archéologiques et monuments historiques est devenue par la suite un guide pour les pillards. Ainsi la destruction spectaculaire de Bamiyan était une exception qui ne doit pas cacher la destruction à petit feu, par le pillage à grande échelle, du patrimoine archéologique afghan.

D'une certaine manière, l'émotion patrimoniale causée par la destruction des Bouddhas de Bamiyan a été aussi spectaculaire que la destruction elle-même. En relayant et en alimentant l'émotion patrimoniale, les médias occidentaux ont fait le jeu des talibans qui voulaient frapper les esprits à l'échelle mondiale. Quelques mois plus tard, à l'automne 2001, ils ont aussi fait le jeu des gouvernements occidentaux qui allaient envahir l'Afghanistan. La destruction des Bouddhas a été un moment charnière, un révélateur de la vulnérabilité du patrimoine. Comme l'ont montré les réactions occidentales, l'émotion patrimoniale est biaisée, mais elle permet aussi de sensibiliser le grand public aux enjeux de la protection du patrimoine.

Le temps passe, mais les images restent, celles de la destruction, mais aussi celles du site au temps de sa splendeur, comme le raconte Bernard Dupaigne :

"Les deux Bouddhas géants ne sont plus qu'un rêve, les fines peintures à sujets religieux ont été martelées, volées ou noircies de fumée; mais la falaise percée de mille grottes garde toujours son pouvoir d'enchantement, changeant de couleur selon l'heure du jour, coiffée d'un manteau de neige en hiver $»^{38}$.

\section{NOTES}

1. Christian Hottin, "Avant-propos ", Livraisons de l'histoire de l'architecture, $\mathrm{n}^{\circ} 17$, juin 2009, p. 9-11.

2. Daniel Fabre, Émotions patrimoniales. Paris, Éditions de la Maison des sciences de l'homme, 2013, $409 \mathrm{p}$.

3. Nathalie Heinich, «Esquisse d'une typologie des émotions patrimoniales" in Émotions patrimoniales, Daniel Fabre (dir.). Paris, Éditions de la Maison des sciences de l'homme, 2013, p. 195-210.

4. Thomas G. Weiss et Nina Connelly, Cultural Cleansing and Mass Atrocities. Protecting Cultural Heritage in Armed Conflict Zones, J. Paul Getty Trust Occasional Papers in Cultural Heritage Policy. $\mathrm{n}^{\circ} 1,2017$.

5. John Darlington, Fake Heritage: Why We Rebuild Monuments, Yale University Press, 2020.

6. Stanislas Julien, Mémoires sur les contrées occidentales, Tome 1, traduits du sanscrit en chinois, en l'an 648 par Hiouen-Tsang; et du chinois en français par M. Stanislas Julien, Paris, Imprimerie impériale, 1857.

7. Le « Grand Jeu ». Le terme a d'abord été utilisé par les diplomates avant d'être popularisé par l'écrivain britannique Rudyard Kipling dans son roman Kim en 1901. 
8. Pierre Centlivres, «Vie, mort et survie des Bouddhas de Bamiyan (Afghanistan) », Livraisons de l'histoire de l'architecture, $\mathrm{n}^{\circ}$ 17, 2009, p. 13-26.

9. Alexander Burnes, "On the Colossal Idols of Bamian", Journal of the Asiatic Society of Bengal, 2, 1833, p. 561-564.

10. Ella Maillart, La voie cruelle, Genève, Éditions Jeheber, 1952.

11. Godard, André, et al., Les antiquités bouddhiques de Bamiyan (Mémoires de la Délégation archéologique française en Afghanistan, tome II), Paris et Amsterdam, G. van Oest, 1928.

12. Pierre Centlivres, «Vie, mort et survie », op. cit.

13. SPACH Newsletter (Society for the Preservation of Afghan Cultural Heritage), 1999.

14. Jean-François Schnoering, "Pourquoi les bouddhas de Bâmiyân ont-ils été détruits?", Bulletin de l'Institut Pierre Renouvin, vol. 31, décembre 2010, p. 127-140.

15. Bakhtar News (agence de presse des talibans), cité dans Pierre Centlivres, Les Bouddhas d'Afghanistan. L'épopée des archéologues, la Croisière jaune à Bâmiyân, la découverte d'un site fabuleux, théâtre de siècles d'aventures et d'histoire, Lausanne, Éditions Favre, 2001, p. 14.

16. Alors poursuivi pour avoir organisé les attentats du 7 août 1998 contre les ambassades américaines du Kenya et de Tanzanie.

17. «Les talibans annoncent avoir "attaqué » militairement les bouddhas de Bamiyan", Le Monde, 2 mars 2001.

18. «La destruction des bouddhas, c'est la fin de tout espoir ", Le Monde, 16 mars 2001.

19. «Les ruines des bouddhas géants de Bamiyan au cœur d'une région ravagée par les combats ", Le Monde, 27 mars 2001.

20. Pierre Lafrance, "Comment les bouddhas de Bamyan n'ont pas été sauvés", Critique internationale, vol. 12, $\mathrm{n}^{\circ}$ 3, 2001, p. 14-21.

21. Barbara Crossette, “Taliban Explains Buddha Demolition”, The New York Times, 2001 : « Quand vos enfants meurent sous vos yeux, alors vous vous fichez d'une œuvre d'art. Si vous détruisez notre avenir avec des sanctions économiques, vous ne pouvez pas vous intéresser à notre patrimoine. Si nous avions voulu détruire ces statues, nous l'aurions fait il y a trois ans. Alors pourquoi ne l'avons-nous pas fait? Dans notre religion, s'il y a quelque chose d'inoffensif, on le laisse. Si l'argent va à des statues alors que des enfants meurent de malnutrition à côté, alors ça devient dangereux, et on le détruit ». Traduction personnelle.

22. Nasir Behzad et Daud Qarizadah, BBC, 2015 : «Je l'ai regretté à l'époque, je le regrette aujourd'hui et je le regretterai toujours. Mais je n'ai pas pu résister, je n'ai pas eu le choix, ils m'auraient tué ». Traduction personnelle.

23. Antoine Leblanc, «La conservation des ruines traumatiques, un marqueur ambigu de l'histoire urbaine ", L'Espace géographique, Tome 39, n 3, septembre 2010, p. 253-266.

24. Patrick Sabatier, "Au musée Guimet, toute la richesse d'un art rescapé », Libération, $1^{\mathrm{er}}$ mars 2002.

25. Fredrik Hiebert et Pierre Cambon (dir.), Afghanistan. Hidden Treasures from the National Museum, Kabul, National Geographic, 2008, 304 p.

26. "In pictures: 3D return for Bamiyan Buddha destroyed by Taliban", BBC, 9 mars 2021.

27. Hélène Joffe, «Le pouvoir de l'image: persuasion, émotion et identification », Diogène, vol. 217, n 1, septembre 2007, p. 102-105.

28. Thomas Barfield, Afghanistan. A Cultural and Political History, Princeton University Press, 2010 : « Le 11 septembre 2001, l'Afghanistan devint soudain important ». Traduction personnelle.

29. Daniel Schlumberger, "L'hellénisme en Afghanistan», Daniel Schlumberger. L'Occident à la rencontre de l'Orient, Mathilde Gelin (dir.), Presses de l'Ifpo, 2010, p. 393-419.

30. Site de l'UNESCO, https://whc.unesco.org/fr/apropos/.

31. Pierre Centlivres, "The Controversy over the Buddhas of Bamiyan", South Asia Multidisciplinary Academic Journal, n², 2, Association pour la Recherche sur l'Asie du Sud, 2008.

32. Pierre Centlivres, ibid. 
33. Abdul Wasey Feroozi, "The Impact of War upon Afghanistan's Cultural Heritage", Archaeological Institute of America, 3 janvier 2004.

34. Omar Khan Massoudi, "Introduction", in Fredrik Hiebert et Pierre Cambon(dir.), Afghanistan. Hidden Treasures from the National Museum, Kabul, National Geographic, 2008, 304 p.

35. Roland Besenval, «Les années noires du patrimoine archéologique d'Afghanistan (1980-2001) », Cahiers d'Asie centrale, n²1/22, Institut Français d'Études sur l'Asie centrale, septembre 2013, p. 69-91.

36. Index archéologique d'Afghanistan, Oxford University Press.

37. Abdul Wasey Feroozi, "The Impact of War", op. cit.

38. Bernard Dupaigne, Afghanistan, monuments millénaires, Imprimerie nationale Éditions, 2007.

\section{RÉSUMÉS}

Cet article s'appuie sur des recherches menées en 2020-2021 à la Faculté d'histoire de l'université d'Oxford. Il propose une réflexion sur l'ambiguïté de l'émotion patrimoniale suscitée dans les pays occidentaux par la destruction des Bouddhas de Bamiyan en mars 2001. Les talibans ont mis en scène cette destruction spectaculaire pour véhiculer un message politique et idéologique à l'échelle mondiale. Vingt ans après, de nouveaux éléments d'analyse, en termes de relations de pouvoir notamment, permettent de mieux comprendre le sens d'une destruction ancrée dans la mémoire collective de toute une génération.

This article relies on research carried out in 2020-2021 at the Faculty of History at Oxford University. It explores the ambiguity of the heritage emotion aroused in Western countries by the destruction of the Buddhas of Bamiyan in March 2001. The Taliban dramatised this spectacular destruction to convey a political and ideological message on a global scale. Twenty years later, new elements of analysis, mainly in terms of power relations, help us to understand the meaning of this destruction which is deeply rooted in the collective memory of a whole generation.

Dieser Beitrag basiert auf Forschungsarbeiten, die in den Jahren 2020-2021 an der Fakultät für Geschichte der Universität Oxford durchgeführt wurden. Er schlägt eine Reflexion über die Ambivalenz der Emotionen vor, die in den westlichen Ländern durch die Zerstörung der BuddhaStatuen von Bamiyan im März 2001 ausgelöst wurden. Die Taliban inszenierten diese spektakuläre Zerstörung, um eine politische und ideologische Botschaft auf globaler Ebene zu vermitteln. Zwanzig Jahre später erlauben uns neue Analyseelemente, insbesondere im Hinblick auf die Machtverhältnisse, die Bedeutung einer Zerstörung, die im kollektiven Gedächtnis einer ganzen Generation verankert ist, besser zu verstehen. 


\section{INDEX}

Mots-clés : Afghanistan, Bouddhas de Bamiyan, émotion patrimoniale, conflits contemporains, opinion publique

Schlüsselwörter : Afghanistan, Buddha-Statuen von Bamiyan, Emotionen zum Kulturerbe, zeitgenössische Konflikte, öffentliche Meinung

Keywords : Afghanistan, Buddhas of Bamiyan, heritage emotion, contemporary conflicts, public opinion

\section{AUTEUR}

\section{PAULINE VERGER}

Élève de l'École normale supérieure à Paris, Pauline Verger est titulaire d'une double licence d'histoire et géographie. Elle est actuellement étudiante du Master 2 de Relations internationales cohabilité par l'Université Paris II Panthéon-Assas et Sorbonne Université. Ses recherches portent sur les liens entre patrimoine culturel et conflits aux $\mathrm{XX}^{\mathrm{e}}$ et $\mathrm{XXI}^{\mathrm{e}}$ siècles. Elle est également engagée au sein de l'association Patrimoine sans frontières pour laquelle elle écrit des articles de sensibilisation sur les grands enjeux du patrimoine. Elle a publié : Pauline Verger, Manon Le Bon, Mathilde Beaufils, Chloé Vincent et Justine Vignères, « Prendre le terrain pour objet : une expérience de recherche collective sur les espaces publics à Ibadan, Nigeria ", Carnets de géographes [En ligne], 14 | 2020 ; article collectif, « Les nouvelles frontières de la défense ", Revue de défense nationale, 2020. Adresse électronique : pauline.verger@ens.psl.eu 